\title{
Respuestas al afecto positivo y ajuste psicológico en la adolescencia
}

\author{
Responses to positive affect and psychological adjustment in adolescence
}

\author{
Diego Gomez-Baya \\ Departamento de Psicología Social, Evolutiva y de la Educación, Universidad de Huelva, España \\ Ramon Mendoza \\ Departamento de Psicología Social, Evolutiva y de la Educación, Universidad de Huelva, España. \\ Susana Paíno \\ Departamento de Psicología Clínica y Experimental, Universidad de Huelva, España \\ Jane E. Gillham \\ Psychology Department, Swarthmore College, Estados Unidos de América
}

Recibido (15 de febrero de 2018) Aceptado (4 de marzo de 2019)

\begin{abstract}
Resumen
La investigación hasta la fecha ha prestado poca atención a la regulación del afecto positivo en la adolescencia. Nuestros objetivos fueron construir un cuestionario para evaluar las respuestas al afecto positivo en adolescentes y estudiar las relaciones que presentan con el ajuste psicológico. Una muestra de 1.810 adolescentes completó el cuestionario de respuestas al afecto positivo (con tres dimensiones: rumiación positiva centrada en la emoción, rumiación positiva centrada en uno mismo e inhibición) y tres autoinformes de ajuste psicológico (satisfacción vital, autoestima y síntomas depresivos). El cuestionario mostró buena fiabilidad por consistencia interna y la misma estructura factorial que estudios previos con adultos. Un modelo de ecuaciones estructurales indicó que las respuestas de rumiación positiva centrada en la emoción y en uno mismo se relacionaron positivamente con la satisfacción vital y la autoestima, y negativamente con los síntomas depresivos, mientras que la inhibición del afecto positivo presentó asociaciones opuestas. Palabras clave: afecto positivo; satisfacción vital; depresión; autoestima; adolescencia.
\end{abstract}

\begin{abstract}
Research to date has paid little attention to positive affect regulation in adolescence. Our aims were to examine psychometric properties of a questionnaire to assess responses to positive affect in adolescents and to study the relationships these responses have with psychological adjustment. A sample of 1,810 adolescents completed the responses to positive affect questionnaire (with three dimensions: emotion-focused and selffocused positive rumination, and dampening) and three self-report measures of psychological adjustment (i.e. life satisfaction, self-esteem and depressive symptoms). The questionnaire had good internal consistency reliability and the same factor structure as previous studies using adult samples. Secondly, a structural equation model showed that emotion-focused and self-focused responses were positively related to life satisfaction and self-esteem, and negatively to depressive symptoms, while dampening presented opposite associations. Keywords: positive affect; life satisfaction; depression; self-esteem; adolescence.
\end{abstract}

Correspondencia: Diego Gomez-Baya, Dirección: Departamento de Psicología Social, Evolutiva y de la Educación, Universidad de Huelva. Avda Fuerzas Armadas s/n. 21007, Huelva (España). Tel.+34 959219208. E-mail: diego.gomez@dpee.uhu.es; Ramon Mendoza-ramon@uhu.es; Susana Paíno-sgpaino@uhu.es; Jane E. Gillham-jgillha1@swarthmore.edu. Conflicto de intereses: Los autores declaran no tener ningún conflicto de intereses. Agradecimientos: Esta investigación fue financiada por la beca de Formación del Profesorado Universitario, del Ministerio de Educación de España (AP2009-4621), otorgada a Diego Gómez-Baya. 


\section{Introducción}

Bajo el término regulación emocional se hace referencia a los procesos mediante los que influimos en las emociones que experimentamos y en cómo y cuándo las expresamos (Gross, 2015). La evidencia hasta la fecha señala que las respuestas a los estados emocionales, más que los estados emocionales en sí mismos, están relacionadas con el ajuste psicológico (Flores \& Delgado, 2015; Mas, Fusté, García-Grau \& Bados, 2015) y con la emergencia y el mantenimiento de los trastornos emocionales (Aldao, Nolen-Hoeksema \& Schweizer, 2010). Por otra parte, se ha documentado un elevado estrés durante la adolescencia, además de un incremento en las conductas de riesgo y una mayor presencia de trastornos psicológicos (Allen \& Sheeber, 2008). Específicamente, se ha detectado un mayor riesgo de aparición de trastornos emocionales, como la depresión (Contreras-Valdez, Hernández-Guzmán \& Freyre, 2015; Jaureguizar, Bernaras, Soroa, Sarasa \& Garaigordobil, 2015). En la mayor parte de los trastornos psicológicos en la adolescencia parecen estar implicadas estrategias disfuncionales de regulación emocional (Balluerka, Aritzeta, Gorostiaga, Gartzia \& Soroa, 2013; Zeman, Cassano, Perry-Parrish \& Stegall, 2006). En esta línea, numerosos estudios basados en la Teoría de los Estilos de Respuesta han mostrado de forma consistente que las personas que producen respuestas rumiativas cuando se sienten tristes tienen una mayor probabilidad de experimentar un incremento en la severidad y en la duración de los síntomas depresivos, mientras que aquellas personas que distraen su atención cuando se sienten tristes presentan un alivio en su sintomatología (Nolen-Hoeksema, Wisco \& Lyubomirsky, 2008). Así, la investigación se ha centrado principalmente en la regulación de los estados afectivos negativos, prestando poca atención a la regulación de los estados afectivos positivos (Bryant \& Veroff, 2007; Quinceno $\&$ Vinaccia, 2014). Algunos trabajos ya han sugerido que la regulación disfuncional de las emociones positivas puede también jugar un importante papel en los trastornos emocionales (Gilbert, Nolen-Hoeksema \& Gruber, 2013).

Según el Modelo Tripartito, la depresión en los niños y adolescentes se caracteriza no sólo por la presencia de afecto negativo generalizado sino también por la anhedonia (Anderson \& Hope, 2008). A este respecto, la anhedonia, uno de los síntomas más comunes en la depresión adolescente, implica una falta o pérdida de interés y de disfrute (Gabbay \& et al., 2015). Gilbert (2012) sugirió que es necesario comprender cómo los adolescentes experimentan y regulan el afecto positivo para explicar mejor el incremento en manifestaciones psicopatológicas durante esta transición evolutiva. La Teoría de la Ampliación y Construcción de Fredrickson (2001) postula que cultivar emociones positi- vas ayuda a construir recursos que aumentan el bienestar e incrementan la probabilidad de volver a sentir emociones positivas de nuevo, además de aumentar la resiliencia ante las emociones negativas (Tugade \& Fredrickson, 2007). De este modo, las estrategias de regulación de las emociones positivas que se aprendan y adopten a lo largo del desarrollo explicarán el posterior bienestar (Bryant, Chadwick \& Kluwe, 2011; Moyano-Díaz, Páez \& Torres, 2016). Recientemente en EEUU, Feldman, Joormann \& Johnson (2008) desarrollaron una escala para evaluar las respuestas al afecto positivo. Esta medida de autoinforme, denominada "Cuestionario de Respuestas al Afecto Positivo", se compone de tres subescalas que miden tres tipos de respuestas al afecto positivo: la rumiación positiva centrada en la emoción, que implica prestar atención y ser más consciente de la experiencia del afecto positivo; la inhibición, que supone reducir la intensidad de la emoción positiva pensando en su condición efímera o en la posibilidad de que aparezcan emociones negativas en el futuro; y la rumiación positiva centrada en uno mismo, que implica relacionar la experiencia del afecto positivo con la propia valía personal o con el logro de metas personales. En la validación de este instrumento con universitarios estadounidenses, Feldman \& et al. (2008) encontraron que la inhibición se relacionaba negativamente con la autoestima y positivamente con los síntomas depresivos. Por otra parte, ambos tipos de rumiación positiva, tanto centrada en la emoción como centrada en uno mismo, se asociaron positivamente con la autoestima y negativamente con los síntomas depresivos. Por su parte, Werner-Seidler, Banks, Dunn \& Moulds (2013) mostraron una asociación negativa entre la rumiación positiva y el síntoma depresivo de anhedonia en un estudio con realizado con una muestra de universitarios australianos. Este instrumento se ha adaptado recientemente a otras lenguas, manteniendo la misma estructura factorial. Raes, Daems, Feldman, Johnson \& Van Gucht (2010) y Bijttebier, Raes, Vasey \& Feldman (2012) construyeron la adaptación belga para muestras de adultos y niños. Además, Olofsson, Boersma, Engh \& Wurm (2014) y Yang \& Guo (2014), adaptaron este cuestionario a muestras de universitarios en Suecia y China, respectivamente.

Muy pocos estudios han investigado por el momento las respuestas al afecto positivo y cómo dichas respuestas se asocian con diferentes indicadores de ajuste psicológico en muestras adolescentes. Con una muestra de niños y adolescentes de entre 10 y 14 años en Bélgica, Bijttebier \& et al. (2012) encontraron que unas bajas puntuaciones en rumiación positiva y altos niveles de estrés predijeron una mayor presencia de síntomas depresivos tras un seguimiento de tres meses. Otro estudio longitudinal en Bélgica halló que una elevada inhibición del afecto positivo predijo una mayor presencia de síntomas depresivos en la adolescencia, 
tras otro seguimiento de tres meses (Raes, Smets, Nelis \& Schoofs, 2012). También las respuestas al afecto positivo pueden afectar a otros aspectos del bienestar, como la satisfacción vital. Quoidbach, Berry, Hansenne \& Mikolajczak (2010), con una muestra de universitarios belgas, encontraron que cuando los participantes experimentaron eventos positivos, focalizar la atención en el momento presente y rumiar positivamente estaba asociado con un incremento en las emociones positivas, mientras que compartir con los demás lo que estaban experimentando se relacionó con un incremento en la satisfacción vital. Por el contrario, no pensar en los eventos positivos que estaban experimentando predijo un descenso en las emociones positivas, y centrar la atención en posibles detalles negativos se asoció con una menor satisfacción vital. En otro estudio, Gentzler, Morey, Palmer \& Yi (2013) analizaron los informes diarios de una muestra de adolescentes estadounidenses de 10 a 14 años, prestando atención a sus reacciones emocionales ante el mejor hecho ocurrido cada día durante una semana. Estos autores apuntaron que aquellos participantes que amplificaron sus respuestas a los eventos positivos celebrando o compartiendo estos eventos con otras personas presentaron un mayor bienestar psicológico que aquellos que minimizaron sus respuestas a los eventos positivos, atribuyendo tales experiencias positivas a factores fuera del propio control o pensando que los sentimientos positivos no durarán mucho tiempo. Además, los adolescentes que minimizaron sus experiencias positivas mostraron más problemas internalizantes y externalizantes.

Hasta la fecha, no se ha validado ninguna escala para medir las respuestas al afecto positivo en adolescentes españoles, siendo la adolescencia una etapa de enorme importancia en el ciclo vital respecto a la emergencia de trastornos afectivos. Por otra parte, muy pocos estudios han analizado las relaciones entre las respuestas al afecto positivo y el ajuste psicológico en la adolescencia, dado que la mayor parte de los estudios se han llevado a cabo con muestras adultas o de universitarios. Además, las investigaciones existentes han analizado especialmente muestras centroeuropeas o norteamericanas, por lo que las respuestas al afecto positico en muestras del sur de Europa no se han estudiado todavía. Gelhaar \& et.al (2007) encontraron diferencias en las estrategias de afrontamiento entre los adolescentes del sur, centro y norte de Europa. De forma específica, Miyamoto \& Ma (2011) subrayaron la importancia de las influencias culturales en las respuestas al afecto positivo. Así, este trabajo de investigación presenta dos objetivos. El primer objetivo consiste en examinar las propiedades psicométricas de un cuestionario para medir las respuestas al afecto positivo en adolescentes españoles. Con este trabajo de investigación, esperamos aportar una medida fiable y válida para la investigación con muestras adolescentes, con una estructura factorial similar a la versión adulta de Feldman \& et al. (2008). El segundo objetivo es analizar las relaciones entre las diferentes respuestas al afecto positivo y variables de ajuste psicológico en la adolescencia, como la autoestima, la satisfacción vital o los síntomas depresivos. Nuestra hipótesis de partida establece que tanto la rumiación positiva centrada en la emoción como la rumiación positiva centrada en uno mismo se relacionan positivamente con la autoestima y la satisfacción vital, y negativamente con los síntomas depresivos, mientras que esperamos que la inhibición del afecto positivo presente asociaciones inversas.

\section{Método}

\section{Participantes}

Un total de 1.810 adolescentes con edades comprendidas entre los 12 y 17 años $(M=14,62, D T=1,32,50,9 \%$ chicas) participaron en esta investigación. Estos adolescentes se encontraban escolarizados en 19 institutos de enseñanza secundaria en Andalucía (España), localizados en diferentes habitats (rural, semiurbano o urbano) y con diferente titularidad (público o concertado). Las clases participantes dentro de cada instituto fueron seleccionadas al azar.

\section{Diseño general del estudio y procedimiento de recogida de datos}

Se ha seguido un diseño transversal, en el que los estudiantes completaron autoinformes de papel y lápiz, de manera individual y anónima en sus respectivas aulas durante una clase normal, tardando alrededor de 30 minutos. Se respetaron todos los principios de la declaración de Helsinki y se obtuvo el consentimiento informado de los participantes y sus padres. Esta investigación obtuvo la aprobación del comité de ética de la Universidad de Huelva (España).

\section{Instrumentos}

Respuestas al afecto positivo. Se administró una versión reducida del Responses to Positive Affect Questionnaire de Feldman y sus colaboradores (2008), que fue adaptado para adolescentes españoles por Gómez-Baya (2014). En dicha adaptación, se realizó una traducción inversa de inglés a castellano del cuestionario original y, tras dos estudios piloto, se redujo el número de items. Esta versión para adolescentes está formada por 12 ítems, distribuidos en 3 dimensiones con el mismo número de ítems, mientras que la escala original para adultos de Feldman \& et.al (2008) está constituida por 17 ítems distribuidos en dimensiones con diferente número de indicadores. El cuestionario usado 
en el presente trabajo de investigación presentó así 12 afirmaciones sobre lo que puedes hacer cuando te sientes feliz o contento/a, que se dividen en tres subescalas, una para la rumiación positiva centrada en la emoción (ítems del 1 al 4: "Pienso en lo feliz que me siento", "Pienso en lo fuerte que me siento", "Pienso en que me siento con fuerzas para hacer de todo" y "Me doy cuenta de que me siento lleno de energía"), otra para la inhibición de afecto positivo (ítems del 5 al 8: "Pienso: Mi racha de suerte va a terminar pronto", "Pienso en cosas que podrían ir mal", "Me recuerdo que estos sentimientos no durarán" y "Pienso: Esto es demasiado bueno para ser verdad"), y otra para la rumiación positiva centrada en uno mismo (ítems del 9 al 12: "Pienso: Lo estoy consiguiendo todo", "Pienso: Estoy alcanzando todo cuanto puedo conseguir", "Pienso en lo orgulloso que me siento de mí mismo" y "Pienso: Lo estoy logrando todo"). Para cada enunciado se proponen cuatro opciones de respuesta sobre la frecuencia con la se realizan las acciones descritas: casi nunca, algunas veces, a menudo, o casi siempre. Estas opciones son puntuadas, en este orden, de 1 a 4. Para calcular la puntuación global en cada subescala, se sumó la puntuación en cada uno de los respectivos cuatro ítems, alcanzando un valor entre 4 y 16.

Autoestima. Se administró la Rosenberg Self-esteem Scale (1965), adaptada al castellano por Salgado \& Iglesias (1995). Esta escala se compone de 10 ítems que presentan una escala de respuesta tipo Likert con 4 opciones desde totalmente de acuerdo a totalmente en desacuerdo.

Satisfacción vital global. Se empleó la adaptación al castellano de la Satisfaction with Life Scale (Atienza, Pons, Balaguer \& García-Mérita, 2000; Diener, Emmons, Larsen \& Griffin, 1985). Está formada por 5 ítems con una escala de respuesta Likert de cinco opciones, desde totalmente en desacuerdo a totalmente de acuerdo.

Síntomas depresivos. Se administró la validación en castellano del Short Children's Depression Inventory (Del Barrio, Roa, Olmedo \& Colodrón, 2002; Kovacs, 2001). Este inventario breve está formado por 10 indicadores y evalúa la presencia e intensidad de síntomas depresivos en niños y adolescentes, siendo válido para su uso en investigación en lugar de la versión extensa.

\section{Diseño de análisis de datos}

Se aplicó la prueba de Little para comprobar que los valores perdidos se distribuyen completamente al azar. Se usó un nivel $\alpha$ de 0,05 para todos los análisis estadísticos. Para estudiar las propiedades psicométricas del cuestionario de Respuestas al afecto positivo en adolescentes, comenzamos examinando los estadísticos descriptivos de los ítems y subescalas. Después se llevó a cabo la prueba KolmogorovSmirnov de normalidad con la corrección de Lilliefors para determinar si los ítems y subescalas se distribuyen siguiendo una curva normal o no paramétrica. En primer lugar, se realizaron correlaciones bivariadas entre los ítems, por un lado, y las escalas, por otro. Se estudió la fiabilidad por consistencia interna de cada subescala, calculando el valor $\alpha$ de Cronbach. En segundo lugar, se exploraron las diferencias de género y de edad en cada dimensión. Estos análisis se llevaron a cabo con el programa estadístico spss 21.0. En tercer lugar, se realizó un análisis factorial confirmatorio para determinar si el cuestionario de Respuestas al afecto positivo presenta una estructura factorial similar a la medida original para adultos. Tras un análisis factorial exploratorio de la estructura del cuestionario, se puso a prueba un modelo en el que los cuatro primeros ítems formaron parte del factor de rumiación positiva centrada en la emoción, el segundo grupo de cuatro ítems compusieron el factor de inhibición, y los últimos cuatro indicadores formaron parte del factor de rumiación positiva centrada en uno mismo. El estadístico $\chi^{2}$ y los índices CFI, BBNFI, RMSEA y el intervalo de confianza (IC) del $90 \%$ de RMSEA fueron calculados para comprobar el ajuste global.

Respecto al segundo objetivo, se realizaron dos tipos de análisis para estudiar las relaciones entre las dimensiones de respuestas al afecto positivo y las variables de ajuste psicológico (satisfacción vital, autoestima y síntomas depresivos). Por un lado, se examinaron las correlaciones bivariadas $\mathrm{y}$, por otro lado, se puso a prueba un modelo de ecuaciones estructurales. Para analizar el ajuste del modelo, se examinaron los indicadores de ajuste global, los residuos estandarizados y las ecuaciones de medida y de constructo. Tanto el modelo de ecuaciones estructurales como el análisis factorial confirmatorio previo fueron realizados con el programa EQS 9.1.

\section{Resultados}

\section{Estadísticos descriptivos}

La prueba de Little indicó que los valores perdidos estaban distribuidos completamente al azar, $\chi^{2}(77, N=1810)=$ $64,72, p=, 840$. Se realizó un procedimiento de estimación por máxima verosimilitud, basado en el algoritmo EM, para imputar los valores perdidos. La Tabla 1 muestra los estadísticos descriptivos de los items y de las dimensiones de respuestas al afecto positivo. Las medias más elevadas se encontraron en los indicadores de rumiación positiva centrada en la emoción, mientras que las más bajas se hallaron en los indicadores de inhibición. El cálculo de cada dimensión de respuestas al afecto positivo se realizó sumando las puntuaciones en los ítems respectivos. 
Tabla 1. Estadísticos descriptivos de los items y de las dimensiones de respuestas al afecto positivo y de las variables de ajuste psicológico.

\begin{tabular}{|c|c|c|c|c|c|c|}
\hline & & Media & Mediana & Desviación Típica & Asimetría & Curtosis \\
\hline \multirow{4}{*}{$\begin{array}{l}\text { Rumiación } \\
\text { Positiva Centrada } \\
\text { en la Emoción }\end{array}$} & $\begin{array}{l}\text { i1. Pienso en lo feliz que me } \\
\text { siento. }\end{array}$ & 3,11 & 3 & ,97 &,- 76 & -.56 \\
\hline & $\begin{array}{l}\text { i2. Pienso en lo fuerte que me } \\
\text { siento. }\end{array}$ & 2,91 & 3 & 1,02 &,- 47 & -.98 \\
\hline & $\begin{array}{l}\text { i3. Pienso en que me siento } \\
\text { con fuerzas para hacer de } \\
\text { todo. }\end{array}$ & 3,19 & 3 & ,90 &,- 81 & .36 \\
\hline & $\begin{array}{l}\text { i4. Me doy cuenta de que me } \\
\text { siento lleno de energía. }\end{array}$ & 3,27 & 4 &, 87 &,- 92 & -.13 \\
\hline \multirow[t]{4}{*}{ Inhibición } & $\begin{array}{l}\text { i5. Pienso "Mi racha de suerte } \\
\text { va a terminar pronto". }\end{array}$ & 2,05 & 2 & 1,05 & ,60 & -.90 \\
\hline & $\begin{array}{l}\text { i6. Pienso en cosas que } \\
\text { podrían ir mal. }\end{array}$ & 1,95 & 2 & 1,02 &, 71 & -.71 \\
\hline & $\begin{array}{l}\text { i7. Me recuerdo que estos } \\
\text { sentimientos no durarán. }\end{array}$ & 1,90 & 2 & 1,03 &, 81 & -.63 \\
\hline & $\begin{array}{l}\text { i8. Pienso "Esto es demasiado } \\
\text { bueno para ser verdad". }\end{array}$ & 2,20 & 2 & 1,12 &, 43 & -1.20 \\
\hline \multirow{4}{*}{$\begin{array}{l}\text { Rumiación } \\
\text { Positiva Centrada } \\
\text { en Uno Mismo }\end{array}$} & $\begin{array}{l}\text { i9. Pienso "Lo estoy } \\
\text { consiguiendo todo". }\end{array}$ & 2,52 & 2 & 1,026 & 03 & -1.13 \\
\hline & $\begin{array}{l}\text { i10. Pienso "Estoy alcanzando } \\
\text { todo cuanto puedo conseguir". }\end{array}$ & 2,53 & 2 & 1,04 &, 02 & -1.17 \\
\hline & $\begin{array}{l}\text { i11. Pienso en lo orgulloso } \\
\text { que me siento de mí mismo. }\end{array}$ & 2,79 & 3 & 1,03 &,- 33 & -1.08 \\
\hline & $\begin{array}{l}\text { i12. Pienso "Lo estoy } \\
\text { logrando todo". }\end{array}$ & 2,52 & 2 & 1,07 &, 00 & -1.25 \\
\hline \multicolumn{2}{|c|}{$\begin{array}{l}\text { Subescala: Rumiación Positiva Centrada en la } \\
\text { Emoción }\end{array}$} & 12.51 & 13 & 3,16 &,- 60 &,- 58 \\
\hline \multicolumn{2}{|c|}{ Subescala: Inhibición } & 8.09 & 7 & 3,54 & ,68 &,- 57 \\
\hline \multicolumn{2}{|c|}{$\begin{array}{l}\text { Subescala: Rumiación Positiva Centrada en Uno } \\
\text { Mismo }\end{array}$} & 10.37 & 10 & 3,64 &, 01 & $-1,04$ \\
\hline \multicolumn{2}{|l|}{ Autoestima } & 30.54 & 31 & 5,17 &,- 45 &, 08 \\
\hline \multicolumn{2}{|l|}{ Satisfacción Vital } & 18.22 & 19 & 4,51 &,- 65 &, 17 \\
\hline \multicolumn{2}{|c|}{ Síntomas Depresivos } & 3.75 & 3 & 2,90 & 1,33 & 2,45 \\
\hline
\end{tabular}


Tabla 2. Correlaciones de Spearman entre los items del cuestionario de respuestas al afecto positivo en la adolescencia.

\begin{tabular}{|c|c|c|c|c|c|c|c|c|c|c|c|c|}
\hline & i1 & i2 & i3 & i4 & i5 & i6 & i7 & i8 & i9 & i10 & i11 & i12 \\
\hline i1 & 1 & & & & & & & & & & & \\
\hline i2 &, $71 * *$ & 1 & & & & & & & & & & \\
\hline i3 &, $58 * *$ &, $66^{* *}$ & 1 & & & & & & & & & \\
\hline i4 &, $55^{* *}$ &, $55^{* *}$ &, $70 * *$ & 1 & & & & & & & & \\
\hline i5 & 03 & 03 &,- 02 &,- 04 & 1 & & & & & & & \\
\hline i6 &,- 01 &, 01 &,$- 07 * *$ &,$- 10 * *$ &, $57 * *$ & 1 & & & & & & \\
\hline i7 &, 02 &, 03 &,- 03 &,$- 09 * *$ &, $62 * *$ &, $66^{* *}$ & 1 & & & & & \\
\hline i8 &, $08 * *$ &, $06^{*}$ &, 03 &,- 02 &, $52 * *$ &, $52 * *$ &, $60 * *$ & 1 & & & & \\
\hline i9 &, $35^{* *}$ &, $40 * *$ &, $42 * *$ &, $39 * *$ & ,01 &,$- 05^{*}$ & ,02 & ,06* & 1 & & & \\
\hline i10 &, $36 * *$ &, $36^{* *}$ &, $37 * *$ &, $37 * *$ &,- 03 &,- 04 &, 01 &, $05^{*}$ &, $72 * *$ & 1 & & \\
\hline i11 &, $44 * *$ &, $45^{* *}$ &, $46^{* *}$ &, $49 * *$ &,$- 08 * *$ &,$- 11 * *$ &,$- 10 * *$ &,- 03 &, $60 * *$ &, $62 * *$ & 1 & \\
\hline i12 &, $38 * *$ &, $40 * *$ &, $42 * *$ &, $39 * *$ &,$- 07 * *$ &,$- 07 * *$ &,- 05 &, 01 &, $73 * *$ &, $72 * *$ &, $69 * *$ & 1 \\
\hline
\end{tabular}

\section{Correlaciones bivariadas y consistencia interna}

La prueba de Kolmogorov-Smirnov mostró un nivel de significación $p<.001$ para todos los ítems y subescalas de respuestas al afacto positivo, lo que indicó que no siguen una distribución normal y resulta necesario aplicar pruebas estadísticas no paramétricas. La Tabla 2 muestra las correlaciones de Spearman entre los ítems. Los indicadores dentro de cada dimensión de respuesta al afecto positivo (ítems 1-4: rumiación positiva centrada en la emoción; ítems 5-8: inhibición; e ítems 9-12: rumiación positiva centrada en uno mismo) se mostraron alta y positivamente relacionados. Además, los indicadores de la dimensión de rumiación positiva centrada en la emoción estuvieron también relacionados de forma positiva con los indicadores de la dimensión de rumiación positiva centrada en uno mismo.

Todas las subescalas de respuestas al afecto positivo presentaron notable fiabilidad, con valores de $\alpha$ de Cronbach por encima de 0,85 (ver Tabla 3). Por otra parte, las correlaciones de Spearman indicaron que las dimensiones de rumiación positiva, centrada en la emoción y centrada en uno mismo, están asociadas positivamente, mientras que las dimensiones de inhibición y de rumiación positiva centrada en uno mismo se relacionaron negativamente, si bien esta última correlación fue muy pequeña.

\section{Diferencias de edad y género en las respuestas al afecto positivo}

La prueba U de Mann-Whitney indicó diferencias de género significativas en la inhibición de afecto positivo, $U=349,10, p=, 015, r=, 06$, y en la rumiación positiva centrada en uno mismo, $U=325,04, p<, 001, r=, 14$. Así, las chicas inhibieron menos el afecto positivo $(M d n=7)$ y presentaron menos rumiación positiva centrada en sí mismas $(M d n=10)$ que los chicos $(M d n=8$ y $M d n=11$, respectivamente). La prueba $\mathrm{H}$ de Kruskal-Wallis indicó diferences de edad significativas en la rumiación positiva centrada en uno mismo, $\chi^{2}(5)=41,74, p<, 001$. De esta forma, los estudiantes de 17 años presentaron una media de $8,93(D T=3,58)$ en rumiación positiva centrada en uno mismo, mientras que los estudiantes de 12 años alcanzaron una media de 10,86 $(D T=3,52)$.

\section{Análisis factorial exploratorio y confirmatorio}

En primer lugar, se llevó a cabo un análisis de componentes principales. La medida de adecuación muestral de Kaiser-Meyer-Olkin obtuvo un valor de 0,86 , y la prueba de esfericidad de Bartlett resultó significativa, $\chi^{2}(66$, $N=1810)=11299,85, p<, 001$. Los resultados de este aná- 
Tabla 3. Correlaciones de Spearman entre las dimensiones de respuesta al afecto positivo y las variables de ajuste psicológico, indicando los índices de fiabilidad por consistencia interna ( $\alpha$ de Cronbach).

\begin{tabular}{lcccccc}
\hline & 1 & 2 & 3 & 4 & 5 & 6 \\
\hline 1. Rumiación Positiva Centrada en la Emoción & $(, 86)$ & & & & & \\
2. Inhibición &, 01 & $(, 86)$ & & & & \\
3. Rumiación Positiva Centrada en Uno Mismo &, $54^{* *}$ &,$- 06^{*}$ & $(, 90)$ & & & \\
4. Autoestima &, $29^{* *}$ &,$- 34^{* *}$ &, $40^{* *}$ & $(, 82)$ & & \\
5. Satisfacción Vital &, $31^{* *}$ &,$- 23^{* *}$ &, $37^{* *}$ &, $54^{* *}$ & $(, 86)$ & \\
6. Síntomas Depresivos &,$- 26^{* *}$ &, $34^{* *}$ &,$- 38^{* *}$ &,$- 65^{* *}$ &,$- 50^{* *}$ & $(, 77)$ \\
\hline
\end{tabular}

Nota. ${ }^{*} p<, 05 * * p<, 01$

lisis mostraron tres factores con valores propios por encima de 1 (Factor $1=4,54$, Factor 2=2,85, y Factor $3=1,37$ ), que explicaron hasta un $73 \%$ de la varianza de las puntuaciones en la escala. Los ítems se distribuyeron en los tres factores de acuerdo con lo esperado teóricamente (rumiación positiva centrada en la emoción, inhibición y rumiación positiva centrada en uno mismo). El siguiente paso consistió en la construcción de un modelo confirmatorio de la estructura factorial del cuestionario. Dada la distribución no paramétrica de las variables, se emplearon técnicas robustas para calcular el ajuste del modelo. Aunque la probabilidad del estadístico Satorra-Bentler $\chi^{2}(51, N=1810)=445,13$, fue $p<, 001$, este índice de ajuste está muy afectado por el tamaño muestral. Otros indicadores robustos señalaron que el modelo presentan un buen ajuste a los datos (BBNFI $=96$, CFI $=, 96$, RMSEA $=, 07$, IC $90 \%$ RMSEA $=, 06-, 07)$. Además, los residuos estadarizados tuvieron valores muy bajos y las ecuaciones de medida resultaron todas significativas, estando todos los factores significativamente constituidos por sus indicadores observables. La Figura 1 muestra los valores de las soluciones estandarizadas. La saturación de todos los indicadores en sus respectivos factores estuvo por encima de 0,70 . Se encontró además una relación positiva entre los factores de rumiación positiva centrada en la emoción y centrada en uno mismo $(\beta=, 60, p<, 001)$. De esta forma, una mayor frecuencia de rumiación positiva centrada en la emoción estuvo asociada con una mayor frecuencia de rumiación positiva centrada en uno mismo.

\section{Relaciones entre las respuestas al afecto positivo y el ajuste psicológico}

La Tabla 1 muestra los estadísticos descriptivos de las variables de ajuste psicológico. En la Tabla 3, las correlaciones de Spearman indicaron que una mayor rumiación positiva centrada en la emoción y una mayor rumiación positiva centrada en uno mismo se asociaron con una ma- yor autoestima y satisfacción vital, y con menos síntomas depresivos. Un mayor uso de la inhibición se relacionó con una menor autoestima y satisfacción vital, y con más presencia de síntomas depresivos. Las escalas de autoestima, satisfacción vital y síntomas depresivos mostraron asimismo buena fiabilidad por consistencia interna.

Después se puso a prueba un modelo de ecuaciones estructurales para analizar las relaciones entre las respuestas al afecto positivo y las variables de ajuste psicológico. En la Figura 2 puede observarse el modelo desarrollado, con los valores de las soluciones estadarizadas. De nuevo, aunque la probabilidad asociada al estadístico Satorra-Bentler $\chi^{2}(77, N=1810)=463,64$, fue $p<, 001$, se estudiaron otros índices dado que se encuentra afectado por el tamaño muestral. Otros indicadores robustos señalaron que el modelo presentó buen ajuste: $\mathrm{BBNFI}=, 96, \mathrm{CFI}=, 97$, $\mathrm{RMSEA}=, 06, \mathrm{y}$ IC $90 \%$ RMSEA $=, 05-, 06$. Además, los residuos estandarizados fueron muy reducidos (entre $-, 1 \mathrm{y}, 1)$ y todas las ecuaciones de medida fueron significativas para todos los indicadores y factores. Se ha encontrado que el factor de rumiación positiva centrada en la emoción se encuentra positivamente relacionado con el factor de rumiación positiva centrada en uno mismo. Los resultados de este modelo indicaron que los factores de rumiación positiva (tanto centrada en la emocion como en uno mismo) se relacionaron positivamente con la autoestima y la satisfacción vital, y negativamente con los síntomas depresivos. El factor de inhibición del afecto positivo estuvo positivamente relacionado con los síntomas depresivos e inversamente con la autoestima y la satisfacción vital. Por otra parte, la autoestima y la satisfacción vital se mostraron asociadas positivamente, mientras que tanto la autoestima como la satisfacción vital se relacionaron negativamente con los síntomas depresivos. En este modelo, las respuestas al afecto positivo explicaron el $28,8 \%$ de la varianza de la autoestima, el $18,9 \%$ de la satisfacción vital y el $28,5 \%$ de los síntomas depresivos. 
Figura 1. Análisis factorial confirmatorio del cuestionario de Respuestas al afecto positivo en la adolescencia.

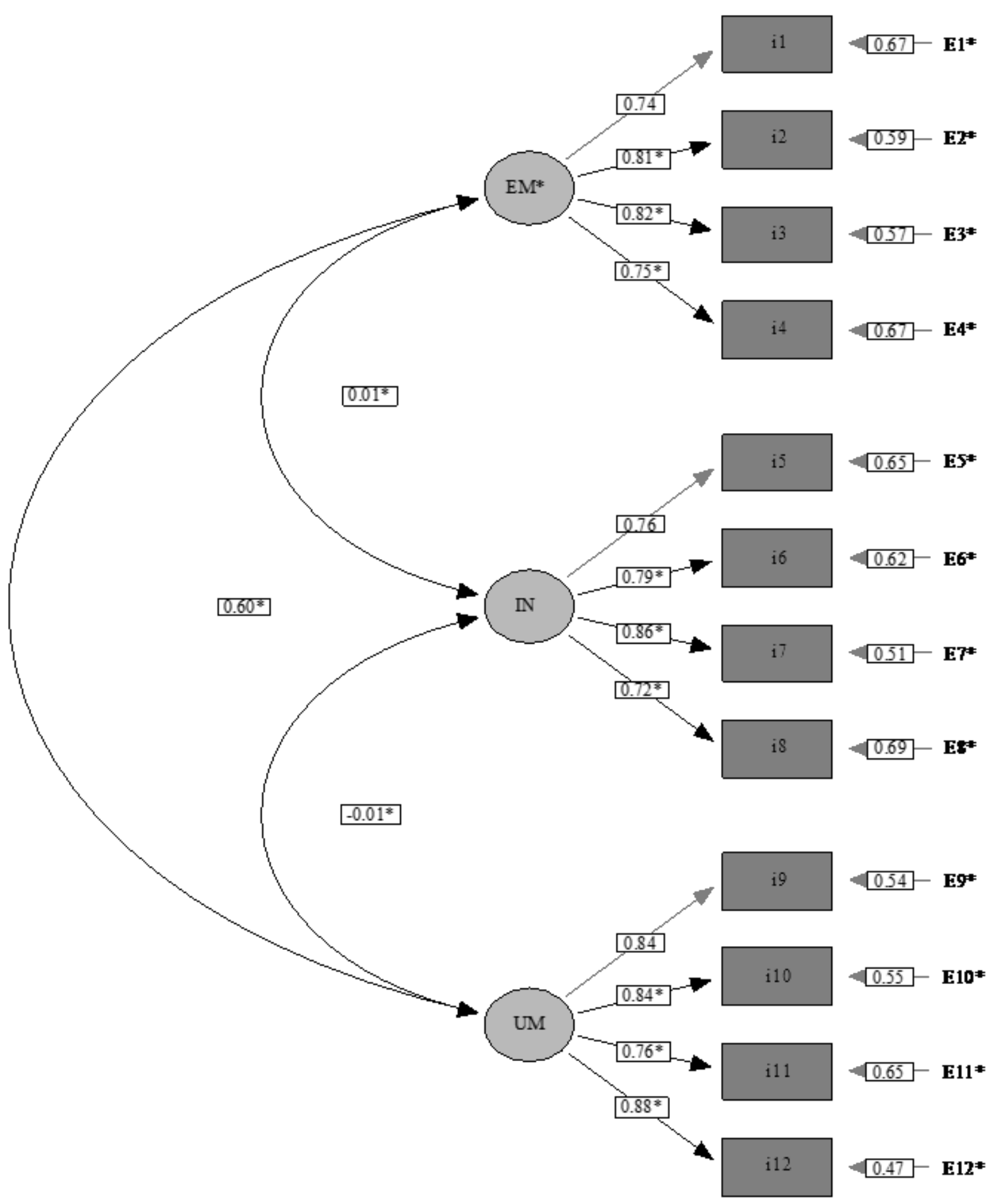

Nota EM= Rumiación positiva centrada en la emoción; IN= Inhibición; UM= Rumiación positiva centrada en uno mismo. 
Figura 2. Modelo de ecuaciones estructurales de las relaciones entre las respuestas al afecto positivo y el ajuste psicológico en la adolescencia.

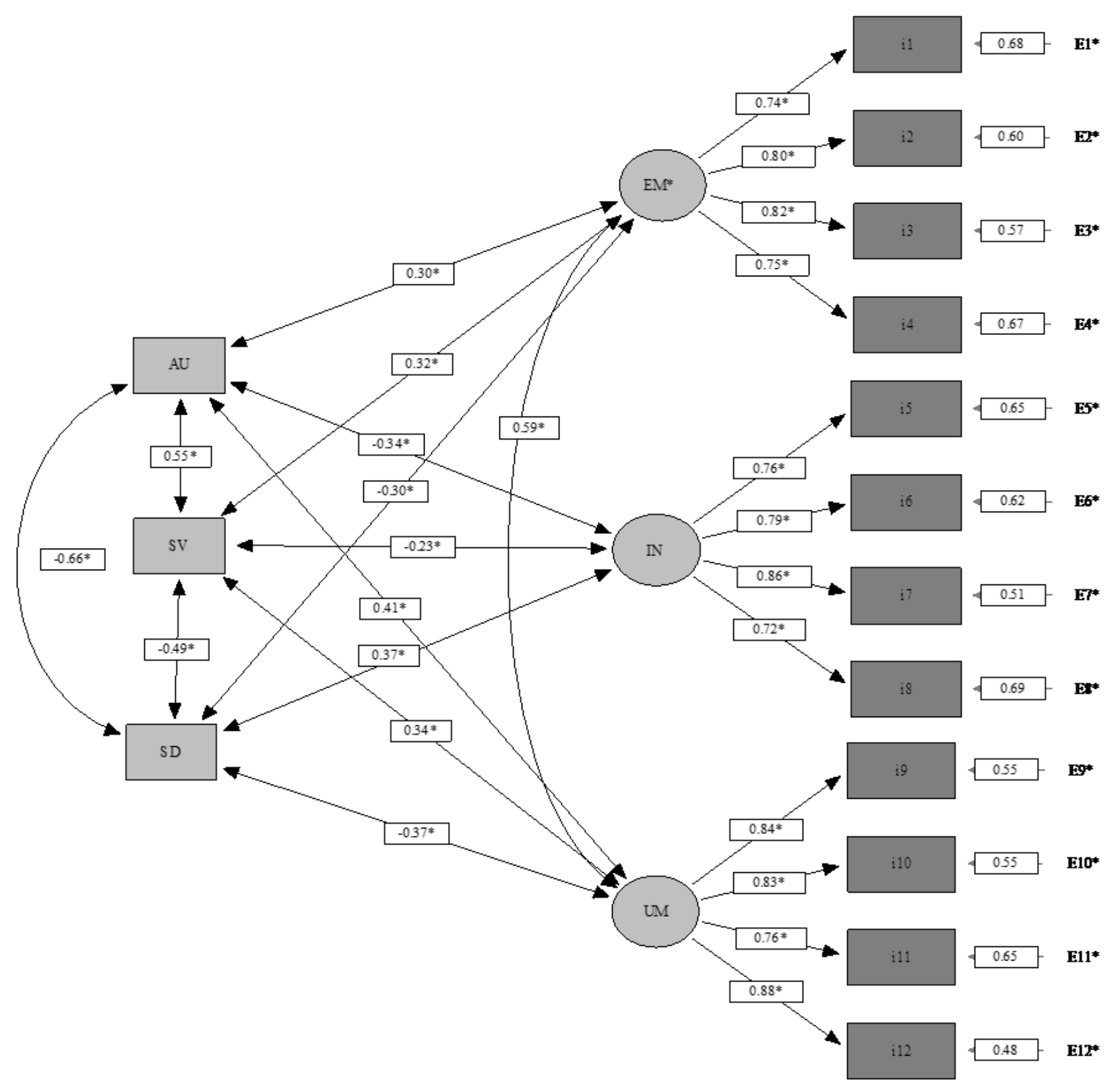

Nota. $\mathrm{EM}=$ Rumiación positiva centrada en la emoción; IN= Inhibición; $\mathrm{UM}=$ Rumiación positiva centrada en uno mismo; $\mathrm{AU}=$ Autoestima; $\mathrm{SV}=$ Satisfacción Vital; SD= Síntomas Depresivos. 


\section{Discusión y conclusiones}

El primer objetivo fue analizar las propiedades psicométricas del cuestionario de respuestas al afecto positivo en adolescentes. Los resultados indicaron, en primer lugar, que los participantes presentaron puntuaciones más elevadas en los indicadores de rumiación positiva centrada en la emoción, y más bajas en los indicadores de inhibición de las emociones positivas. Así, parece que los adolescentes tienden a amplificar más sus emociones positivas que a inhibirlas cuando se sienten felices. En segundo lugar, cada subescala (rumiación positiva centrada en la emoción, inhibición, y rumiación positiva centrada en uno mismo) presentó una notable fiabilidad por consistencia interna. En tercer lugar, se han encontrado diferencias de edad y de género en las respuestas al afecto positivo. Las chicas inhibieron menos el afecto positivo, pero también atribuyeron menos ese afecto positivo a su propia valía o al logro de sus metas personales. Por otra parte, los adolescentes de mayor edad reflejaron una menor rumiación positiva centrada en ellos mismos que los adolescentes de menor edad. Finalmente, un análisis factorial confirmatorio mostró que el cuestionario presenta una adecuada validez factorial. Un modelo con buen ajuste a los datos identificó tres factores de respuestas al afecto positivo (rumiación positiva centrada en la emoción, inhibición, y rumiación positiva centrada en uno mismo), cada uno de ellos compuesto por sus respectivos indicadores. Este modelo señaló también que las respuestas de rumiación positiva, centrada en la emoción y centrada en uno mismo, se encuentran relacionadas positivamente. Por consiguiente, la escala presentó buena fiabilidad por consistencia interna y la misma estructura factorial que las versiones previas con muestras adultas, como el trabajo original de Feldman y et al. (2008) en EEUU o la adaptación belga desarrollada por Raes y et al. (2010). También en la misma línea que el trabajo de Feldman y et al. (2008), las dos subescalas de rumiación positiva se mostraron fuertemente asociadas de forma positiva. Así, en línea con nuestra hipótesis de partida, se ha aportado un instrumento fiable y válido para usar en la investigación con muestras adolescentes.

El segundo objetivo consistió en analizar las relaciones entre las respuestas al afecto positivo y el ajuste psicológico en la adolescencia. Las correlaciones indicaron que una mayor satisfacción vital, una mayor autoestima y una menor presencia de síntomas depresivos están asociadas con una mayor atención y toma de consciencia de las emociones positivas, así como con una mayor atribución de estas emociones a la propia valía y con una menor inhibición o reducción de la experiencia de estas emociones positivas. A partir de estas correlaciones, un modelo de ecuaciones estructurales con buen ajuste a los datos señaló que: a) las respuestas de rumiación positiva centrada en la emoción y en uno mismo estaban relacionadas positivamente con la autoetima y la satisfacción vital, y negativamente con los síntomas depresivos; b) la inhibición se muestra asociada negativamente con la autoestima y la satisfacción vital, pero positivamente con los síntomas depresivos; y c) las respuestas de rumiación positiva se muestran positivamente asociadas, al igual que la autoestima y la satisfacción vital, que a su vez se relacionan negativamente con los síntomas depresivos.

Los resultados hallados respecto a las relaciones entre las respuestas al afecto positivo, la autoestima y la satisfacción vital, son consistentes con nuestra hipótesis y con estudios previos con muestras de universitarios, aunque ningún estudio ha mostrado estos resultados evaluando muestras adolescentes (Feldman \& et al., 2008; Quoidbach \& et al., 2010). Estas asociaciones pueden ser explicadas desde la Teoría de la Ampliación y Construcción de Fredrickson (2001), de manera que prestar mayor atención y tomar una mayor consciencia de la experiencia del afecto positivo incrementaría la satisfacción vital mediante el incremento de la probabilidad de sentir emociones positivas de nuevo, mientras que la rumiación positiva centrada en uno mismo fomentaría la autoestima al permitir una mayor atribución del afecto positivo a la propia valía o al logro de objetivos personales, lo que proporcionaría recursos para construir autopercepciones positivas. Por otra parte, las asociaciones observadas entre las respuestas al afecto positivo y los síntomas depresivos son también consistentes con nuestra hipótesis de partida y con los resultados de estudios previos. Hasta la fecha, sólo dos trabajos han analizado estas relaciones con los síntomas depresivos evaluando muestras adolescentes (Bijttebier \& et al., 2012; Raes \& et al., 2012). Dado que la anhedonia es uno de los síntomas definitorios de la depresión, las respuestas ante el afecto positivo tienen un importante papel en la depresión adolescente. También, siguiendo la Teoría de la Ampliación y Construcción, la inhibición del afecto positivo dificultaría un procesamiento cognitivo adaptativo, estrechando el foco de atención y reduciendo el repertorio de pensamiento-acción, lo que obstaculizaría el bienestar emocional (Fredrickson \& Branigan, 2005). Así, este trabajo de investigación aporta una media concisa, fiable y válida para la investigación sobre las respuestas ante el afecto positivo en adolescentes españoles. Por otra parte, por primera vez se han analizado en una muestra de adolescentes las relaciones que presentan las diferentes respuestas al afecto positivo con varios indicadores de bienestar, como la autoestima, la satisfacción vital y los síntomas depresivos. Tenemos que destacar también que, dadas las diferencias culturales discutidas por Gelhaar y et al. (2007) y Miyamoto \& Ma (2011) en las conductas de afrontamiento y en las respuestas al afecto positivo, este trabajo de investigación aporta la primera evidencia con 
una muestra del sur de Europa, mostrando resultados similares a los detectados con muestras norteamericanas o centroeuropeas.

Aunque esta investigación presenta importantes aportaciones, también deben señalarse algunas limitaciones. Primero, dado que se ha seguido un diseño transversal, nuestras conclusiones sólo pueden basarse en asociaciones entre variables. Resulta necesario pues llevar a cabo una investigación longitudinal que permita analizar cómo las respuestas al afecto positivo predicen el posterior bienestar psicológico del adolescente. Una segunda limitación puede venir del uso de autoinformes con escala de respuesta de tipo Likert, dada la posible imprecisión del informe retrospectivo de los adolescentes sobre sus respuestas tras experimentar emociones positivas. Aunque este instrumento ha mostrado buenas propiedades psicométricas y supone una medida concisa y fácil de administrar en tareas de investigación, pudiera resultar de interés estudiar la validez convergente de este cuestionario comparando sus resultados con informes diarios, siguiendo el procedimiento empleado por Gentzler \& et al. (2013).

Además de las líneas de investigación sugeridas, este estudio puede ofrecer algunas implicaciones prácticas. Los resultados de esta investigación pueden sugerir la puesta en marcha de intervenciones para incrementar las respuestas de rumiación positiva y reducir la frecuencia de inhibición de emociones positivas. Dado que durante la adolescencia existe un mayor riesgo de que aparezcan trastornos emocionales como la depresión, y se ha prestado poca atención hasta la fecha a la regulación de los estados afectivos positivos (Gilbert, 2012), podría resultar de interés el diseño e implementación de programas de promoción del bienestar en las escuelas mediante la construcción de respuestas más adaptativa a los afectos positivos, además de ante los afectos negativos, quizás integrando intervenciones desde la psicología cognitivo-conductual (Horowitz \& Garber, 2006; Stice, Shaw, Bohon, Martin \& Rohde, 2009) y la psicología positiva (Gillham \& et al., 2013). El programa Aulas Felices supone una aplicación de la psicología positiva al ámbito educativo que ha mostrado eficacia para aumentar el bienestar psicológico en las aulas (Arguís, Bolsas, Hernández \& Salvador, 2010). Un buen punto de partida puede estar en la evidencia hallada por Bryant \& Veroff (2007) y por Hurley \& Kwon (2012), que diseñaron numerosas actividades para promover una respuesta más adaptativa al afecto positivo caracterizada por el saboreo de los eventos positivos que ocurren en el día a día. McMakin, Siegle \& Shirk (2011) diseñaron una intervención consistente en escribir sobre una experiencia positiva en el pasado, recordando los detalles específicos y los sentimientos asociados. Esta intervención se mostró eficaz para reducir los síntomas depresivos en los jóvenes.
Quoidbach, Mikolajczak \& Gross (2015) concluyeron que imaginar eventos positivos y desarrollar actividades que permitan las interacciones sociales, el ejercicio físico y el contacto con la naturaleza, incrementan las emociones positivas y el bienestar psicológico a largo plazo. Por otra parte, haber aportado aquí un instrumento con buenas propiedades psicométricas en adolescentes permitiría evaluar si dichas intervenciones han mejorado significativamente el estilo de respuesta al afecto positivo, y de esta forma poder comprender si los cambios en el bienestar psicológico se deben a cambios en los estilos de respuesta ante el afecto positivo.

\section{Referencias}

Aldao, A., Nolen-Hoeksema, S. \& Schweizer, S. (2010). Emotion-regulation strategies across psychopathology: A meta-analytic review. Clinical psychology review, 30, 217-237. doi:10.1016/j.cpr.2009.11.004

Allen, N. B., \& Sheeber, L. B. (2008). Adolescent emotional development and the emergence of depressive disorders. Cambridge: Cambridge University Press.

Anderson, E. R., \& Hope, D. A. (2008). A review of the tripartite model for understanding the link between anxiety and depression in youth. Clinical Psychology Review, 28, 275-287. doi:10.1016/j.cpr.2007.05.004

Arguís, R., Bolsas, A. P., Hernández, S. \& Salvador, M. M. (2010). Programa Aulas Felices. Psicología Positiva aplicada a la educación. Recuperado de http://catedu.es/psicologiapositiva/

Atienza, F. L., Pons, D., Balaguer, I. \& García-Mérita, M. (2000). Propiedades psicométricas de la Escala de Satisfacción con la Vida en adolescentes. Psicothema, 12, 314-319.

Balluerka, N., Aritzeta, A., Gorostiaga, A., Gartzia, L., \& Soroa, G. (2013). Emotional intelligence and depressed mood in adolescence: A multilevel approach. International Journal of Clinical and Health Psychology, 13, 110-117. doi:10.1016/S1697-2600(13)70014-0

Bijttebier, P., Raes, F., Vasey, M. W., \& Feldman, G. C. (2012). Responses to positive affect predict mood symptoms in children under conditions of stress: a prospective study. Journal of abnormal child psychology, 40, 381-389. doi:10.1007/s10802-011-9579-2

Bryant, F. B., Chadwick, E. D., \& Kluwe, K. (2011). Understanding the processes that regulate positive emotional experience: Unsolved problems and future directions for theory and research on savoring. International Journal of Wellbeing, 1, 107-126. doi:10.5502/ijw.v1i1.18

Bryant, F. B., \& Veroff, J. (2007). Savoring: A new model of positive experience. Mahwah, NJ: Lawrence Erlbaum Associates Publishers.

Contreras-Valdez, J. A., Hernández-Guzmán, L., \& Freyre, M. Á. (2015). Validez de constructo del Inventario de Depresión de Beck II para adolescentes. Terapia psicológica, 33, 195-203. http://dx.doi. org/10.4067/S0718-48082015000300004

Del Barrio, V., Roa, M. L., Olmedo, M. \& Colodrón, F. (2002). Primera adaptación del CDI-s a población española. Acción Psicológica, 3, 263-272. doi:http://dx.doi.org/10.5944/ap.1.3.558 
Diener, E. D., Emmons, R. A., Larsen, R. J. \& Griffin, S. (1985). The satisfaction with life scale. Journal of personality assessment, 49, 71-75. doi:10.1207/s15327752jpa4901_13

Feldman, G. C., Joormann, J. \& Johnson, S. L. (2008). Responses to positive affect: A self-report measure of rumination and dampening. Cognitive Therapy and Research, 32, 507-525. doi:10.1007/s10608-006-9083-0

Flores, M. D. C. R., \& Delgado, A. O. (2015). De la competencia emocional a la autoestima y satisfacción vital en adolescentes. Psicología Conductual, 23, 345-359.

Fredrickson, B. L. (2001). The role of positive emotions in positive psychology: The broaden-and-build theory of positive emotions. American psychologist, 56, 218-226. doi:10.1037/0003-066X.56.3.218

Fredrickson, B. L. \& Branigan, C. (2005). Positive emotions broaden the scope of attention and thought-action repertoires. Cognition \& emotion, 19, 313-332. doi:10.1080/02699930441000238

Gabbay, V., Johnson. A. R., Alonso, C. M., Evans, L. K., Babb, J. S. y Klein, R. G. (2015). Anhedonia, but not irritability, is associated with illness severity outcomes in adolescent major depression. Journal of Child and Adolescent Psychopharmacology, 25, 194-200. doi:10.1089/ cap.2014.0105

Gelhaar, T., Seiffge-Krenke, I., Borge, A., Cicognani, E., Cunha, M., Loncaric, D., Macek, P., Steinhausen, H.-C. \& Metzke, C. W. (2007). Adolescent coping with everyday stressors: A seven-nation study of youth from central, eastern, southern, and northern Europe. European Journal of Developmental Psychology, 4, 129-156. doi:10.1080/17405620600831564

Gentzler, A. L., Morey, J. N., Palmer, C. A. \& Yi, C. Y. (2013). Young Adolescents' Responses to Positive Events: Associations With Positive Affect and Adjustment. The Journal of Early Adolescence, 33, 663-683. doi:10.1177/0272431612462629

Gilbert, K. E. (2012). The neglected role of positive emotion in adolescent psychopathology. Clinical psychology review, 32, 467-481. doi:10.1016/j.cpr.2012.05.005

Gilbert, K. E., Nolen-Hoeksema, S. \& Gruber, J. (2013). Positive emotion dysregulation across mood disorders: How amplifying versus dampening predicts emotional reactivity and illness course. Behaviour research and therapy, 51, 736-741. doi:10.1016/j.brat.2013.08.004

Gillham, J.E., Abenavoli, R.M., Brunwasser, S.B., Linkins, M., Reivich, K.J. \& Seligman, M.E.P. (2013). Resilience Education. En S.A. David, I. Boniwell, y A.C. Ayers (Eds.) Handbook of Happiness. (pp. 609-630). Oxford University Press.

Gómez-Baya, D. (2014). Predictores de la satisfacción vital y de los sintomas depresivos en la adolescencia (Tesis doctoral, Universidad de Huelva, España). Recuperado de http://rabida.uhu.es/dspace/ handle/10272/7981.

Gross, J. J. (2015). Emotion regulation: Current status and future prospects. Psychological Inquiry, 26, 1-26. doi:10.1080/1047840X.2014.940781

Horowitz, J. L. \& Garber, J. (2006). The prevention of depressive symptoms in children and adolescents: A meta-analytic review. Journal of consulting and clinical psychology, 74, 401-415. http://dx.doi. org/10.1037/0022-006X.74.3.401
Hurley, D. B., \& Kwon, P. (2012). Results of a study to increase savoring the moment: Differential impact on positive and negative outcomes. Journal of Happiness Studies, 13, 579-588. doi: 10.1007/s10902-011-9280-8

Jaureguizar, J., Bernaras, E., Soroa, M., Sarasa, M., \& Garaigordobil, M. (2015). Sintomatología depresiva en adolescentes y variables asociadas al contexto escolar y clínico. Psicología Conductual, 23, 245-264.

Kovacs, M. (2001). Children's Depression Inventory manual. North Tonawanda. New York: Multi-Health Systems.

Mas, N., Fusté, A., García-Grau, E., \& Bados, A. (2015). Coping styles and vulnerability to eating disorders in adolescent girls, by age. Terapia psicologica, 33, 161-168. http://dx.doi.org/10.4067/ S0718-48082015000300001

McMakin, D. L., Siegle, G. J., \& Shirk, S. R. (2011). Positive Affect Stimulation and Sustainment (PASS) module for depressed mood: A preliminary investigation of treatment-related effects. Cognitive Therapy and Research, 35, 217-226. doi:10.1007/s10608-010-9311-5

Miyamoto, Y., \& Ma, X. (2011). Dampening or savoring positive emotions: A dialectical cultural script guides emotion regulation. Emotion, 11, 1346-1357. doi:10.1037/a0025135

Moyano-Díaz, E., Páez, D., \& Torres, M. (2016). Propiedades psicométricas del cuestionario para medir estrategias de aumento de la felicidad (HIS) en versión castellana (CEA-EAP). Terapia psicológica, 34, 143-154. http://dx.doi.org/10.4067/S0718-48082016000200006

Nolen-Hoeksema, S., Wisco, B. E., \& Lyubomirsky, S. (2008). Rethinking rumination. Perspectives on psychological science, 3, 400-424. doi:10.1111/j.1745-6924.2008.00088.x

Olofsson, M. E., Boersma, K., Engh, J., \& Wurm, M. (2014). A psychometric evaluation of the Swedish version of the Responses to Positive Affect questionnaire. Nordic journal of psychiatry, 68, 588-593. doi $: 10.3109 / 08039488.2014 .898792$

Quiceno, J. M., \& Vinaccia, S. (2014). Calidad de vida en adolescentes: Análisis desde las fortalezas personales y las emociones negativas. Terapia psicológica, 32, 185-200. http://dx.doi.org/10.4067/ S0718-48082014000300002

Quoidbach, J., Berry, E. V., Hansenne, M., \& Mikolajczak, M. (2010). Positive emotion regulation and well-being: Comparing the impact of eight savoring and dampening strategies. Personality and Individual Differences, 49, 368-373. doi:10.1016/j.paid.2010.03.048

Quoidbach, J., Mikolajczak, M., \& Gross, J. J. (2015). Positive interventions: An emotion regulation perspective. Psychological bulletin, 141, 655-693. doi:http://dx.doi.org/10.1037/a0038648

Raes, F., Daems, K., Feldman, G., Johnson, S., \& Van Gucht, D. (2010). A Psychometric Evaluation of the Dutch Version of the Responses to Positive Affect Questionnaire. Psychologica Belgica. 49, pp.293-310. doi:http://doi.org/10.5334/pb-49-4-293

Raes, F., Smets, J., Nelis, S., \& Schoofs, H. (2012). Dampening of positive affect prospectively predicts depressive symptoms in non-clinical samples. Cognition \& Emotion, 26, 75-82. doi:10.1080/02699931.2 011.555474

Rosenberg, M. (1965). Society and the adolescent self-image. Princeton, NJ: Princeton University Press. 
Salgado, J., \& Iglesias, M. (1995). Estructura de la Escala de Autoestima de Rosenberg: Un análisis factorial confirmatorio. Psicológica: Revista de metodología y psicología experimental, 16, 441-454.

Stice, E., Shaw, H., Bohon, C., Marti, C. N., \& Rohde, P. (2009). A meta-analytic review of depression prevention programs for children and adolescents: factors that predict magnitude of intervention effects. Journal of consulting and clinical psychology, 77, 486-503. http://dx.doi. org/10.1037/a0015168

Tugade, M. M., \& Fredrickson, B. L. (2007). Regulation of positive emotions: Emotion regulation strategies that promote resilience. Journal of Happiness Studies, 8, 311-333. doi:10.1007/s10902-006-9015-4

Werner-Seidler, A., Banks, R., Dunn, B. D., \& Moulds, M. L. (2013). An investigation of the relationship between positive affect regulation and depression. Behaviour research and therapy, 51, 46-56. doi:10.1016/j. brat.2012.11.001

Yang, H., \& Guo, W. (2014). Chinese version of the responses to positive affect questionnaire: testing the factor structure, reliability, and validity in a college student sample. Psychological reports, 115, 467-484. doi:10.2466/08.21.PR0.115c22z8

Zeman, J., Cassano, M., Perry-Parrish, C., \& Stegall, S. (2006). Emotion regulation in children and adolescents. Journal of Developmental \& Behavioral Pediatrics, 27, 155-168. doi:10.1097/00004703-20060400000014 